\title{
PROMOÇÃO DA SAÚDE: CONCEPÇÕES E PRÁTICAS DOS ENFERMEIROS DA ESTRATÉGIA SAÚDE DA FAMÍLIA
}

\author{
HEALTH PROMOTION: CONCEPTIONS AND PRACTICES OF THE NURSES OF THE FAMILY \\ HEALTH STRATEGY
}

DOI: $10.16891 / 2317-434 X . v 8 . e 2 \cdot a 2020 . p p 509-517$

Recebido em: 20.02.2020 | Aceito em: 09.04.2020 João Paulo Xavier Silva", Ana Paula Ribeiro de Castroa, Crisângela Santos de Meloa, Raphaely de
Sousa Feitosaa, Ariadne Gomes Patrício Sampaioa, Ana Maria Machado Borges

Centro Universitário Dr. Leão Sampaioa

E-mail: joaopauloxavier@leaosampaio.edu.br

\begin{abstract}
RESUMO
O Sistema Único de Saúde é potencial no reconhecimento das necessidades populacionais em suas múltiplas especificidades, principalmente por meio da Estratégia Saúde da Família. Nesse contexto, o enfermeiro é figura de importante atuação na promoção da saúde, pois atua de maneira dinâmica e integrada em todas as atividades desenvolvidas nas Unidades Básicas de Saúde. O presente estudo tem como objetivo avaliar as concepções e práticas dos enfermeiros da Estratégia Saúde da Família no que concerne à Promoção da Saúde. Trata-se de uma pesquisa com abordagem qualitativa, do tipo descritiva e exploratória, de corte transversal. Participaram treze enfermeiros e a coleta de dados foi realizada por meio de uma entrevista semiestruturada. A análise dos dados esteve pautada na análise do tipo categorial temática proposta por Minayo. O estudo obteve aprovação do Comitê de Ética em Pesquisa para sua realização. Emergiram quatro categorias de acordo com os núcleos de sentido identificados nas falas dos enfermeiros, estas serviram para agrupar os relatos que apresentaram pressupostos temáticos semelhantes. Foram estes: O conhecimento dos enfermeiros sobre promoção da saúde; A formação dos enfermeiros como influenciadora na promoção da saúde; As ações de promoção da saúde desenvolvidas pelos enfermeiros; e O empoderamento e a coparticipação dos usuários como estratégia de promoção da saúde. Os discursos, por mais elaborados que sejam, revelam concepções fragilizadas, o que pode interferir negativamente na prática profissional. Evidenciam-se ainda atividades de caráter individual, de cunho curativista, sendo insuficientes e incompatíveis com a práxis da promoção da saúde em suas amplas dimensões.
\end{abstract}

Palavras-chave: Promoção da saúde; Enfermeiro; Estratégia Saúde da Família.

\section{ABSTRACT}

The Unified Health System is potential in the recognition of population needs in its multiple specificities, mainly through the Family Health Strategy. In this context, the nurse is an important figure in the Health Promotion because it acts in a dynamic and integrated way in all the activities developed in the Basic Health Units. The present study aims to evaluate the conceptions and practices of nurses of the Family Health Strategy regarding Health Promotion. This is a qualitative, descriptive and exploratory research with a cross-sectional approach. Thirteen nurses participated and the data collection was performed through a semistructured interview. Data analysis was based on thematic categorical type analysis proposed by Minayo. The study was approved by the Research Ethics Committee for its accomplishment. Four categories emerged according to the nuclei of meaning identified in nurses' statements, which served to group the reports that presented similar thematic assumptions. These were: Nurses' knowledge about health promotion; The training of nurses as na influencer in health promotion; Health promotion actions developed by nurses; and Empowerment and the sharing of users as a health promotion strategy. The discourses, however elaborate they may be, reveal fragile conceptions, which can negatively interfere with professional practice. There are also activities of an individual character, curativist, being insufficient and incompatible with the praxis of health promotion in its broad dimensions.

Keyword: Health promotion; Nurse; Family Health Strategy. 


\section{INTRODUÇÃO}

A Promoção da saúde desponta internacionalmente na década de 1970 a partir de uma nova concepção de saúde que considera não somente a dimensão biológica ao se pensar em saúde, mas reconhece a existência de determinantes sociais e econômicos que constroem uma compreensão ampla de saúde não centrada exclusivamente na doença. Surge, assim, uma perspectiva contra hegemônica dos modelos assistenciais e organizacionais até então puramente configurados nos sistemas de saúde (PINHEIRO, et al., 2018).

Nos últimos anos, o Brasil tem investido na formulação e implementação de políticas públicas de promoção da saúde, buscando construir progressivamente um modelo de atenção que privilegie uma abordagem assistencial voltada para a melhoria da qualidade de vida em coletividade (PINHEIRO, et al., 2018; FERREIRA; PÉRICO; DIAS, 2018).

É nesse contexto, que o Sistema Único de Saúde (SUS) se torna potencial no reconhecimento das necessidades populacionais em suas múltiplas especificidades, principalmente por meio da Estratégia Saúde da Família (ESF) e da Política Nacional de Promoção da Saúde (PNPS). A ESF propõe uma nova visão de saúde nos processos de intervenção da atenção primária e nas ações de promoção da saúde. A organização dessa estratégia é preconizada por um modelo que rompe com o molde da assistência médica individual e age em conjunto com os indivíduos de forma integral e contínua (FERREIRA; PÉRICO; DIAS, 2018).

O enfermeiro é figura importante de atuação na promoção da saúde, pois atua de maneira dinâmica e integrada em todas as atividades prestadas nas Unidades Básicas de Saúde (UBS). Na perspectiva da promoção da saúde, os cuidados de enfermagem objetivam desenvolver nos indivíduos a capacidade de identificar as suas necessidades e participar conjuntamente na busca de soluções para elas, considerando seus limites e possibilidades. Em complemento, a literatura especializada considera as atividades de enfermagem no campo da atenção primária em saúde como fundamentais para prover mudanças nas ações de saúde e direcionar as práticas à promoção da saúde (COLLIÉRE, 2013).

Considerando essas prerrogativas, questionase: Quais as concepções e práticas dos enfermeiros atuantes na ESF no que concerne à Promoção da Saúde? Esta investigação torna-se relevante ao possibilitar uma análise de como se fundamenta a práxis do enfermeiro na Atenção Primária, sua relação e aplicabilidade com a promoção da saúde. Contribuindo assim para uma reorientação profissional na reorganização dos serviços prestados à comunidade com vistas à qualidade de vida e bem-estar propostos pela Carta de Ottawa.

Frente ao exposto, o estudo teve como objetivo avaliar as concepções e práticas dos enfermeiros da Estratégia Saúde da Família no que concerne à Promoção da Saúde.

\section{MÉTODO}

Trata-se de uma pesquisa de abordagem qualitativa, do tipo descritiva e exploratória, de corte transversal. Esse delineamento permite uma análise crítica e interpretativa a partir das concepções e práticas dos sujeitos do estudo sobre a promoção da saúde. Desenvolveu-se nas Unidades Básicas de Saúde (UBS) da Estratégia Saúde da Família (ESF), na zona urbana do município de Juazeiro do Norte, Ceará.

A coleta de dados foi realizada durante os meses de março, abril e maio de 2016. Participaram desse estudo treze enfermeiros atuantes nas UBS que foram aleatoriamente selecionadas como campo de estudo. A amostragem utilizada para a coleta de dados foi do tipo não probabilística, por acessibilidade ou conveniência. $\mathrm{O}$ número de participantes foi definido com base na saturação teórica, ou seja, pelo início de repetição das informações relatadas e pela ausência de novas informações sobe o fenômeno estudado.

Para participação da pesquisa, os enfermeiros enquadraram-se nos seguintes critérios de inclusão: enfermeiros de ambos os sexos; possuir atuação na ESF há pelo menos seis meses. Os critérios de exclusão foram: estar de licença, férias ou greve; estar ausente do serviço no período da coleta dos dados.

A coleta de dados foi realizada por meio de entrevistas semiestruturadas e áudio gravadas, constituídas por quatro perguntas abertas, utilizando-se de um roteiro previamente elaborado, apresentado no Quadro 1 a seguir. As entrevistas foram realizadas no local de trabalho dos participantes, após agendamento prévio, sendo a duração média de 15 a 30 minutos. Salienta-se que previamente se aplicou um pré-teste para avaliação do roteiro da entrevista, do qual participaram 5 (cinco) interlocutores-chave, não havendo necessidade de ajustes no instrumento de coleta.

A análise dos dados teve como referencial metodológico a análise do tipo categorial temática, sistematizada por Minayo (2014). Dessa forma, ordenaram-se as três fases essenciais do método: 1) préanálise, na qual se deu a constituição do corpus e a leitura flutuante; 2) exploração de material, na qual consistiu em 
uma operação classificatória para compreensão textual; e 3) tratamento dos resultados, na qual se realizou a interpretação e se estabeleceram as associações teóricas e contextuais com outros estudos.

Nesta pesquisa foi assegurado o cumprimento das normas para a pesquisa com seres humanos presentes nas Resoluções 466/12 e 510/16 do Conselho Nacional de Saúde. Para a garantia do anonimato foi alterado o nome dos participantes da pesquisa, atribuindo-lhes nomes de flores. Salienta-se a utilização do Termo de Consentimento Livre e Esclarecido (TCLE) para todos os participantes, a obtenção da anuência pela Secretaria Municipal de Saúde (SESAU) do município de Juazeiro do Norte, e a apreciação ética favorável emitida pelo Comitê de Ética em Pesquisa (CEP) sob o parecer $n^{\circ}$ 1.248.671.

Quadro 1. Roteiro temático para entrevista

\begin{tabular}{|c|l|}
\hline $\mathbf{0 1}$ & O que você entende em relação à Promoção da Saúde? \\
\hline $\mathbf{0 2}$ & $\begin{array}{l}\text { Você já participou de algum curso ou formação que contemplasse essa temática? Se sim, poderia me } \\
\text { relatar como foi essa experiência? }\end{array}$ \\
\hline $\mathbf{0 3}$ & $\begin{array}{l}\text { Você realiza em sua rotina profissional na ESF ações de saúde que possam vir a promover saúde na } \\
\text { comunidade? Se sim, poderia citar e relatar como ocorre? }\end{array}$ \\
\hline $\mathbf{0 4}$ & $\begin{array}{l}\text { De que forma você identifica o empoderamento do usuário e a sua coparticipação no processo de } \\
\text { cuidado em saúde? }\end{array}$ \\
\hline
\end{tabular}

\section{RESULTADOS E DISCUSSÃO}

\section{Caracterização dos sujeitos}

Para melhor compreensão dos sujeitos do estudo, faz-se necessário uma caracterização de acordo com as seguintes variáveis: sexo, idade, tempo de formação acadêmica, tempo de atuação na ESF e titulação.

Em relação ao gênero, a prevalência foi feminina, com doze enfermeiras e somente um profissional do sexo masculino. O predomínio do sexo feminino é confirmado por pesquisa anterior realizada pelo Conselho Federal de Enfermagem e pela Fundação Oswaldo Cruz, a qual aponta que $84,6 \%$ dos profissionais da enfermagem do Brasil são mulheres. Esse dado também é justificado pelo modelo nightingaleano, marcado historicamente pela feminilização da profissão (MACHADO et al., 2017).

No que se refere à idade, verifica-se que os enfermeiros entrevistados se encontravam na faixa etária entre 27 e 55 anos de idade. Esses resultados convergem com estudos prévios realizados no Rio de Janeiro e em Cuiabá, nos quais se evidenciou uma faixa etária semelhante de enfermeiros atuantes na ESF, que se estabeleceu entre 28 e 54 anos de idade (FARIA;
ACIOLI; GALLASCH, 2016; ÁUREA et al., 2012).

No tocante ao tempo de formação acadêmica, houve uma variação significativa. Encontraram-se enfermeiros que possuíam entre dois a 26 anos de formação acadêmica. Esses dados nos levam às reflexões acerca de um novo campo de trabalho para a enfermagem, o que possibilita entender que a experiência adquirida com a idade não é mais um aspecto fundamental para a atuação profissional. O tempo de atuação profissional está interligado às habilidades, atitudes e competências necessárias no ambiente de trabalho (LIMA, 2018).

Quanto à titulação, verificou-se que somente uma enfermeira possuía pós-graduação strictu sensu, sendo mestrado profissional em Saúde da Família. Os outros doze entrevistados declararam titulação de pósgraduação lato senso. Dentre estes, sete são especialistas em Saúde da Família, quatro são especialistas em Políticas Públicas em Saúde Coletiva e um especialista em Saúde Pública. Salienta-se que sete enfermeiros também declararam o título de especialista em áreas distintas à atuação na ESF, dentre as quais estão Auditoria em Saúde; Centro Cirúrgico e Central de Material Esterilizado; Urgência e Emergência; e Docência no Ensino Superior.

Identifica-se que a maioria dos enfermeiros 
que se dedicam ao trabalho assistencial, mais especificamente na atenção básica, limitam-se a formação em cursos de especialização. Alguns fatores contribuem para essa realidade, como a impossibilidade de afastamento do trabalho, os gastos extras com estudos, a necessidade de licença e a falta de apoio dos gestores de saúde municipais (ÁUREA et al., 2012.; LIMA, 2018).

\section{Categorização das falas dos sujeitos}

Emergiram quatro categorias de acordo com os núcleos de sentido identificados nos discursos dos enfermeiros, estas serviram para agrupar os relatos que apresentaram pressupostos temáticos semelhantes. As categorias temáticas foram: 1) $\mathrm{O}$ conhecimento dos enfermeiros sobre promoção da saúde; 2) A formação dos enfermeiros como influenciadora na promoção da saúde; 3) As ações de promoção da saúde desenvolvidas pelos enfermeiros; 4) $\mathrm{O}$ empoderamento e a coparticipação dos usuários na ESF como estratégia de promoção da saúde.

Sequencialmente, constitui-se a categorização temática dos dados obtidos pelas entrevistas, tendo em vista facilitar a análise, apresentação e discussão das informações. Nessa perspectiva, se estabeleceu um diálogo articulado e comparativo entre os dados desta pesquisa e os referenciais teóricos pertinentes a esse estudo, como também com outros estudos semelhantes.

\section{Categoria 1 - O conhecimento dos enfermeiros sobre promoção da saúde}

Nos relatos, dez entrevistados compreendem a promoção da saúde em seu significado amplo e fazem associação à qualidade de vida dos indivíduos. Estes elementos estão contidos nas falas a seguir:

Promoção da saúde é um conjunto de aspectos relacionados ao bem-estar do indivíduo, considerando não apenas a condição saúde, mas moradia, lazer, trabalho. Então, é um conjunto de aspectos relacionados à pessoa e à coletividade (Alfazema).

É uma estratégia de empoderamento voltada para indivíduos e coletividades com o objetivo de melhorar a sua qualidade de vida, e consequentemente o nível de saúde, e torná-los capazes de se auto cuidar (Margarida).

Mesmo com discursos que buscam conceituar a promoção da saúde em sua amplitude, destaca-se que os enfermeiros pouco expressaram elementos indispensáveis à promoção da saúde como o "empoderamento", a autonomia e a educação, apresentando conceituações fragilizadas e incompletas (ALMEIDA; BITTAR, 2015).

Outras falas tornam explícita a má compreensão do termo "promoção da saúde". Dois enfermeiros pautaram seus conceitos no modelo tradicional de prevenção das doenças, relacionando a promoção à prevenção como se assumissem o mesmo significado, conforme visualizado nos relatos:

A promoção é a prevenção, tentando evitar os danos, mas também melhorar a saúde daquele paciente que já está acometido. (Camélia)

Promover é termos que trabalhar em cima de evitar a doença. Promoção é prevenir, né? (Gardênia)

Na prevenção, o indivíduo passa a ser alvo de intervenção no intuito de evitar a doença ou a enfermidade. No âmbito da promoção ocorre um processo participativo e contínuo, não voltado exclusivamente às patologias ou aos danos, mas direcionado para transformações que englobam o estilo de vida.

Esse equívoco expresso pelos enfermeiros aproxima-se de outro estudo no qual para os profissionais de enfermagem a prevenção é considerada um elemento-chave para a promoção da saúde, mas que parece não existir o distanciamento da compreensão entre prevenção e promoção (FERREIRA; PÉRICO; DIAS, 2018). O modelo preventivo tem o seu papel dentro da promoção da saúde, mas se considerado isoladamente é pouco efetivo diante da complexidade do processo saúde-doença.

Vale destacar que essa confusão não se trata somente de uma questão conceitual, mas também operacional. Apesar dos termos promoção e prevenção estarem ligados aos cuidados com a saúde, a prevenção propõe o estabelecimento de estratégias que venham a minimizar ou controlar riscos do adoecer, enquanto a promoção reconhece situações que favoreçam a qualidade de vida e o bem-estar (CZERESNIA; FREITAS, 2003).

Percebe-se a necessidade de uma maior profundidade teórica na compreensão dos enfermeiros sobre promoção da saúde. Tal fragilidade pode interferir na qualidade da assistência, sendo necessário apropriarse conceitualmente do termo para desenvolver atividades verdadeiramente promotoras de saúde. 
Categoria 2 - A formação dos enfermeiros como influenciadora na promoção da saúde

É de extrema importância a formação continuada dos enfermeiros atuantes na ESF, a fim de proporcionar discussões e práticas que sejam contributivas com uma aproximação maior entre as ações de enfermagem e a promoção da saúde. Nesse contexto, apresentam-se as Competências em promoção da saúde (CompHP) como intuito de estabelecer bases teóricas em um sistema próprio na formação conduzida para a promoção da saúde (PINHEIRO et al., 2015).

Ao serem questionados sobre a sua formação como influenciadora na promoção da saúde, levando em consideração a participação em cursos, a leitura de artigos, textos, manuais do Ministério da Saúde (MS), dentre outras atividades, nove dos enfermeiros relataram as suas experiências prévias voltadas à temática. As falas a seguir apontam para essa interpretação:

...em alguns congressos que eu participei sempre existiam palestras direcionadas a esse tema. E também na pós-graduação que eu fiz... que foi em saúde pública, lá eu paguei um módulo direcionado a promoção da saúde e foi uma grande experiência. (Begônia)

Os cursos que realizei foram de grande importância para minha atuação na promoção da saúde na ESF, pois permitiram atualização dos conhecimentos e abriram caminhos para um novo olhar na realização de atividades voltadas a promoção e educação em saúde. (Margarida)

Os relatos permitem inferir que a participação em cursos voltados para a promoção da saúde é um relevante fator na formação desses enfermeiros. Percebese a satisfação com as experiências vividas e os benefícios que tais processos trouxeram para a prática profissional na assistência à saúde. Apesar de existirem inúmeros congressos e cursos na temática referida, notase ainda que não houve participação efetiva dos profissionais em programas de educação continuada promovida por gestores de saúde, pois por vezes a própria gestão não promove ou estimula a integração dos profissionais nesse tipo de atividade.

Para o CompHP, o conjunto de competências necessárias para ações eficazes em promoção da saúde deve abranger valores (equidade, justiça, ética e autonomia); habilidades (princípios conceituais) e conhecimentos. Estes, estruturam-se em uma rede de domínios, a saber: advocacia em saúde, comunicação, liderança, planejamento, parceria, avaliação e pesquisa.
Em conjunto, fornecem as diretrizes para as habilidades e competências em promoção da saúde (PINHEIRO et al., 2015). Salienta-se que esses aspectos não foram abordados satisfatoriamente nos discursos, o que aponta para uma lacuna entre as proposições teóricas do CompHP e a sua aplicabilidade na prática cotidiana.

Vale ressaltar que três entrevistados foram enfáticos ao negar a participação prévia em cursos direcionados à promoção da saúde, ou não recordaram se já haviam participado. Os trechos a seguir correspondem a essa afirmação:

Na verdade, eu não lembro se eu participei. Mas assim, durante a faculdade a gente deve ter trabalhado com isso... (Camélia)

\section{Não, não participei. (Girassol)}

Não. Nunca realizei um curso com essa temática. (Dália)

A aparente insuficiência no que concerne à participação em processos formativos põe em questão a necessidade da continuidade do processo ensinoaprendizagem entre profissionais de saúde. Todavia, essa fragilidade se configura como um obstáculo para a incorporação da promoção da saúde na rotina assistencial dos enfermeiros. Desenvolve-se, assim, a caracterização de uma crise que causa uma série de desordens, pois gera um caminhar incerto e contraditório na assistência à saúde por enfermeiros pouco qualificados (ROECKER; BUDÓ; MARCON, 2012).

Ao investigar sobre os desafios para a promoção da saúde no contexto da formação do enfermeiro, outros autores também denunciam que uma considerável quantidade dos participantes da sua pesquisa não retratou significativamente a complexa rede das relações entre as possibilidades de se promover saúde e a dinâmica das práticas por eles executadas. Este dado revela a falta de entusiasmo perante novos processos formativos e o comodismo de suas ações de acordo com os modelos tradicionais e biomédicos de atenção à saúde (TAVARES et al., 2016).

\section{Categoria 3 - As ações de promoção da saúde} desenvolvidas pelos enfermeiros

A Política Nacional de Promoção da Saúde (PNPS) foi institucionalizada no Brasil em 2006, sofrendo revisões no ano de 2014 (BRASIL, 2006; BRASIL, 2014). A política define estratégias principais para o desenvolvimento da promoção da saúde e sua 
concretização na prática profissional, de modo que se convertam benefícios na sociedade. Para isso, coloca-se a necessidade do delineamento de ações no campo da saúde que sejam capazes de apontar para a resolução dos problemas complexos vivenciados pelos indivíduos (ALMEIDA; BITTAR, 2015).

$O$ enfermeiro que atua na ESF deve ser capacitado para realizar atividades promotoras da saúde, reconhecendo as necessidades, realidades e subjetividades da comunidade atendida em seu território (PINHEIRO et al., 2015). Quando questionados sobre quais atividades desenvolvidas com enfoque na promoção da saúde na comunidade, emergiram os seguintes discursos:

Educação em saúde para gestantes, grupo de puericultura, temos o grupo "Viver com saúde", também trabalho com terapias alternativas... Faço muito a integração ensino serviço com esses grupos. Organizamos brindes, cafés da manhã e são diferenciais, pois atraem a população a participar ativamente. Combino tudo com a comunidade! (Bromélia)

As atividades que têm sido desenvolvidas durante esse ano são as rodas de conversa sobre alimentação saudável e grupo de atividade física. (Rosa)

Nota-se que as atividades realizadas para a promoção da saúde são principalmente a composição de grupos em saúde, palestras educativas, rodas de conversa, dinâmicas e eventos de confraternização.

Para promover saúde na comunidade o enfermeiro deve trabalhar sob a ótica da educação em saúde, incidindo sobre as condições de vida e peculiaridades daquela população e favorecendo uma abordagem crítico reflexiva em coletividade. Nesse cenário, a educação em saúde é um instrumento efetivamente transformador, no sentido da formação ética e ideológica de sujeitos livres que têm potencialidades para produção de saberes e transmissão de conhecimentos (GALAVOTE et al., 2016).

Percebe-se que os grupos de saúde e as rodas de conversa são momentos cruciais para ações de promoção da saúde, pois permeiam a comunicação horizontal entre os interlocutores. Salienta-se que a participação ativa da comunidade durante as atividades desenvolvidas pelos enfermeiros só foi descrita uma vez. Apesar de os entrevistados relatarem suas ações como favoráveis a promoção da saúde, pouco explicitaram em seu discurso o protagonismo social, o que faz parecer que a comunidade é coadjuvante durante a realização de tais atividades.

É nessa vertente que a literatura internacional tem empreendido esforços na construção de uma nova proposta para as ações educativas com vistas à promoção da saúde. Assim, a Organização Mundial da Saúde (OMS) define a Literacia em Saúde como o conjunto de competências cognitivas e sociais e a capacidade dos indivíduos para compreenderem e usarem a informação de formas que promovam e mantenham boa saúde. Considera-se, dessa forma, que todos os sujeitos envolvidos, seja profissionais ou usuários dos serviços de saúde, atuem como protagonistas desse processo (QUEMELO et al., 2017).

Outros relatos revelam que ainda existe uma prática meramente assistencialista, hierarquizada e falha por parte de alguns profissionais. Os trechos a seguir demonstram essa afirmação:

\section{... a gente tenta orientar em relação às condições de saúde com atividades preventivas. (Alfazema)}

Realizo atividades individuais no consultório de enfermagem... (Margarida)

A assistência de enfermagem concretiza-se sob o modelo clínico e individual quando a finalidade é a recuperação de um corpo doente, compreendendo assim uma ação instrumental do cuidar na ótica curativista. Nessa perspectiva, não há espaço para a consolidação da promoção da saúde, pois o cuidado prestado assume um caráter transversal e desinteressado pela real transformação do processo saúde-doença dos sujeitos (ROECKER; BUDÓ; MARCON, 2012).

Assim, cabe ressaltar que a assistência holística não se resume a palestras sistematizadas e ações verticalizadas. Para prestar uma assistência holística, não se pode acreditar na capacidade de fazê-la individualmente. Consultas de cunho curativista, orientações individuais e palestras sistematizadas não são ações favoráveis à promoção e saúde, pois apenas refletem práticas engessadas e metódicas que não trazem contribuições significativas aos sujeitos envolvidos (GALAVOTE et al., 2016).

\section{Categoria 4 - $O$ empoderamento e a coparticipação dos usuários como estratégia de promoção da saúde}

O vínculo é o resultado das relações interpessoais, do cliente e enfermeiro, capaz de transformar realidades e propiciar melhorias de vida na 
comunidade. É instituído nessas relações a coparticipação e responsabilização pela saúde, propiciando o empoderamento como estratégia para a melhora da qualidade de vida dos usuários (LIMA et al., 2014).

É necessário transformar as práticas de saúde a partir de mudanças no processo de inteiração dos sujeitos envolvidos no trabalho em saúde. A proposta da promoção da saúde apenas é possível com o protagonismo simultâneo de profissionais e usuários. Estabelece-se o sentido da corresponsabilidade no cuidado em saúde (CZERESNIA; FREITAS, 2003; TAVARES et al., 2016).

Nessa perspectiva, os enfermeiros foram questionados sobre suas atividades como possíveis fomentadoras do empoderamento aos usuários da ESF, como também lhes foi indagado sobre a identificação da coparticipação desses usuários no processo de promoção da saúde. As respostas apontam para os seguintes horizontes:

O empoderamento se estabelece através da troca de conhecimento, sempre procuro trabalhar que a saúde é direito de todos e dever do estado. Então você tem que dar o poder à comunidade para que eles saibam que é um direito deles. A coparticipação deve existir em todos os momentos, eu parto do princípio que a educação é uma troca de informação. É uma construção que fazemos juntos e todos tem suas responsabilidades, por meio de uma parceria com eles isso acontece naturalmente. (Bromélia)

A partir do momento que ele tem consciência... ele tem poder e autonomia sobre seus direitos $e$ deveres na saúde. (Anemona)

A questão dos direitos e deveres dos usuários nos serviços de saúde foi a mais perceptível nos discursos, o que nos leva a identificar uma preocupação dos enfermeiros em esclarecer aos usuários que todos devem assumir uma postura de cidadania e compromisso. Em contribuição, a Carta dos direitos dos usuários da saúde apresenta princípios básicos de cidadania que asseguram os direitos ao ingresso nos serviços de saúde, sendo uma importante ferramenta de sensibilização social, fonte de informação para os profissionais e usuários (HEIDEMMAN; WOSNY; BOEHS, 2014).

Acerca da coparticipação dos usuários, foi unânime o relato de que ela é identificada em todos os momentos e está intrinsecamente relacionada à autonomia. Esse fato permite evidenciar a reciprocidade como um fator indispensável na promoção da saúde.

Em estudos sobre as necessidades de saúde e a autonomia dos usuários na ESF como fator desencadeador da coparticipação, os resultados convergem com os desta pesquisa, nos quais se compreendeu que os enfermeiros são conscientes da necessidade de uma participação ativa e do envolvimento dos sujeitos no trabalho em saúde na atenção básica de maneira autônoma e voluntária (DURAND; HEIDEMMAN, 2013; LOPES, 2015).

Todavia, também emergiram discursos que demonstram a má compreensão dos enfermeiros sobre o empoderamento, considerando tal processo como individual e transversal. Os trechos comprovam essa afirmativa:

O empoderamento é mais a questão do paciente ter a consciência daquilo que lhe faz bem ou lhe faz mal. É respeitar a decisão do paciente... (Alfazema)

O empoderamento é percebido quando o paciente tem o poder de decidir se vai ou não aderir aquilo. (Girassol)

As falas demonstram uma compreensão superficial ao se abordar temática complexa e multifacetada. O empoderamento vai além da consciência individual e não pode ser avaliado por meio de méritos rasos e simplistas, considerando apenas determinados critérios, como afirmação ou negação e bem ou mal.

Os múltiplos sentidos do empoderamento lhe conferem distintos campos do conhecimento. Dentre esses, incluem-se a ideologia de ação social, os direitos civis, a postura cidadã, a educação em saúde etc. Para explicitar o empoderamento é preciso buscar diversas questões contextualizadas com as mudanças individuais e coletivas, com a ressignificação ideológica, com as estruturas de poder existentes e com as relações internas entre profissionais e usuários dos serviços de saúde (LOPES, 2015).

Tais postulações direcionam para reflexões sobre o empoderamento como quesito primordial para o alcance da promoção da saúde. Sua efetivação é necessária para a melhoria das condições de vida dos indivíduos, a partir do momento que lhes concede a capacidade de mediar conflitos e solucionar problemas em saúde (SOUZA et al., 2014). 
Os enfermeiros da ESF possuem méritos fundamentais para o trabalho focado na promoção da saúde dos sujeitos. Compreender o empoderamento e a coparticipação dos usuários como essenciais para a promoção da saúde é uma das formas de potencializar as próprias ações cotidianas e refletir acerca das estratégias utilizadas para se promover saúde.

\section{CONSIDERAÇÕES FINAIS}

Ao avaliarmos as concepções e práticas dos enfermeiros da ESF no que concerne à promoção da saúde, observamos que estas são delineadas em duas vertentes, sendo a primeira com enfoque no modelo biomédico curativista, e a outra sobre o adequado significado de promoção da saúde, conforme preconizado pela Carta de Ottawa.

Os resultados dessa pesquisa evidenciam a realização de ações desenvolvidas com vistas à promoção da saúde pautadas em atividades grupais, palestras educativas, dinâmicas e rodas de conversa, o que corrobora com os preceitos de promoção da saúde sugeridos pela PNPS e pelas estratégias de aprendizagem significativa.

No entanto, verificou-se uma ausência de relatos sobre a participação ativa da comunidade, transparecendo que, no contexto da promoção da saúde, os enfermeiros assumem o papel de protagonistas. Assim, percebe-se a necessidade de uma práxis mais elaborada sobre o empoderamento e a coparticipação nos processos assistenciais em saúde a partir de uma reflexão que potencialize o desenvolvimento de indivíduos verdadeiramente empoderados e transformadores de sua realidade.

O trabalho aponta como sugestão a realização de investigações que elucidem outros aspectos relacionados à essa temática no contexto da Atenção Primária à Saúde, considerando também as concepções e práticas dos usuários dos serviços e da equipe multiprofissional que o compõe.

\section{REFERÊNCIAS}

ALMEIDA, M.A.; BITTAR, C.M.L. Concepções sobre promoção da saúde por enfermeiros de uma cidade do interior de São Paulo. Brasilian Journal of Research, n.17, v.2, p. 45-51, 2015.

DOI: https://doi.org/10.21722/rbps.v17i2.13187

ÁUREA, C.P.C. et al. Sociodemographic and professional profile of primary health care nurses in Cuiabá - Mato Grosso. Rev. Elet. Enferm. n. 14, v.1, p. 171-180, 2012. DOI: https://doi.org/10.1590/S1414-81452010000200022

BRASIL, Ministério da Saúde. Política Nacional de Promoção da Saúde. Portaria ${ }^{\circ} 687$ MS/GM, de 30 de março de 2006. Aprova a Política de Promoção da Saúde. Diário Oficial da União, 2006.

BRASIL, Ministério da Saúde. Política Nacional de Promoção da Saúde. Portaria no 2.446 MS/GM, de 11 de novembro de 2014. Redefine a Política Nacional de Promoção da Saúde. Diário Oficial da União, 2014.

CZERESNIA, D.F.; FREITAS, C.M. Promoção da saúde: conceitos, reflexões, tendências. Rio de Janeiro: Fiocruz, 2003.

COLLIÈRE, M.F. Cuidar... A primeira arte da vida. Loures: Lusociência, 2013.
DURAND, M.K.; HEIDEMMAN, I.T.S.B. Promoção da autonomia da mulher na consulta de enfermagem em saúde da família. Rev Esc Enferm USP, n. 47, v.2, p.288-95. 2013. DOI: https://doi.org/10.1590/S0080-62342013000200003

FARIA, M.G.A.; ACIOLI, S.; GALLASCH, C.H. Perfil de enfermeiros fluminenses da Estratégia Saúde da Família participantes de um curso de especialização. Rev Enferm. Foco, n. 1, v.1, p.52-55, 2016. DOI: https://doi.org/10.21675/2357-707X.2016.v7.n1.667

FERREIRA, S.R.S.; PÉRICO, L.A.D.; DIAS, V.R.G.F. The complexity of the work of nurses in Primary Health Care. Rev. Bras. Enferm, n.71, v.1, p.752-757. 2018. DOI: http://dx.doi.org/10.1590/0034-7167-2017-0471

GALAVOTE, H.S. et al. The nurse's work in primary health care. Esc Anna Nery Rev Enferm, n. 20, v. 1, p. 90-98. 2016. DOI: http://dx.doi.org/10.5935/1414-8145.20160013

HEIDEMMAN, I.T.S.B.; WOSNY, A.M.; BOEHS, A.E. Health promotion in primary care: study based on the Paulo Freire method. Ciência \& Saúde Coletiva, n.19, v.8, p.35533559. 2014. DOI: https://doi.org/10.1590/1413$\underline{81232014198.11342013}$

LIMA, C.A. et al. Relação profissional-usuário de saúde da 
família: perspectiva da bioética contratualista. Rev. bioét. $\mathrm{n}$. 22, v.1, p. 152-160. 2014. DOI: https://doi.org/10.1590/S1983-80422014000100017

LIMA, F. R.O. A identidade Profissional da Enfermeira na Estratégia Saúde da Família.

(Dissertação de Mestrado). Universidade Federal da Bahia, Salvador, 2018. Acesso em: 15 de setembro de 2016. Disponível em: https://repositorio.ufba.br/ri/handle/ri/25998

LOPES, A.A.F. Cuidado e Empoderamento: a construção do sujeito responsável por sua saúde na experiência do diabete. Saúde Soc, n. 24, v.2, p. 486-500. 2015. DOI: http://dx.doi.org10.1590/S0104-12902015000200008

MACHADO, M.H. et al. Relatório final da Pesquisa Perfil da Enfermagem no Brasil (Convênio: Fiocruz/COFEN). [Internet]. Version 1. Rio de Janeiro, Brasil: NERHUSDAPSENSP/FIOCRUZ e COFEN, 750p. 2017.

MINAYO, M. C. S. O desafio do conhecimento. $14^{\text {a }}$ ed. São Paulo: Hucitec, 2014.

PINHEIRO, D.C.M. et al. Competências em promoção da saúde: desafios da formação. Saúde Soc. n. 24, v.1, p. 180188. 2015. DOI: https://doi.org/10.1590/S010412902015000100014
PINHEIRO, F.T.S. et al. Reflexões sobre o Sistema Único de Saúde: da gênese à crise contemporânea. Rev Sanare, n.17, v.2, p. 82-90. 2018.

DOI: https://doi.org/10.36925/sanare.v17i2.1265

QUEMELO, P.R.V. et al. Literacia em saúde: tradução e validação de instrumento para pesquisa em promoção da saúde no Brasil. Cad. Saúde Pública, n. 22, v.2. 2017. DOI: https://doi.org/10.1590/0102-311x00179715

ROECKER, S.; BUDÓ, M.L.D.; MARCON, S.S. Trabalho educativo do enfermeiro na Estratégia Saúde da Família: dificuldades e perspectivas de mudanças. Rev. Esc. Enferm. n. 46, v.3, p. 641-649. 2012. DOI: https://doi.org/10.1590/S0080-62342012000300016

SOUZA, J.M. et al. Aplicabilidade prática do empowerment nas estratégias de promoção da saúde. Ciência \& Saúde Coletiva, n. 19, v.7. 2014. DOI: https://doi.org/10.1590/1413-81232014197.10272013

TAVARES, M.F.L. et al. A promoção da saúde no ensino profissional: desafios na saúde e a necessidade de alcançar outros setores. Ciência \& Saúde Coletiva, n. 21, v.6, p. 1799-1808. 2016. DOI: https://doi.org/10.1590/1413$\underline{81232015216.07622016}$ 